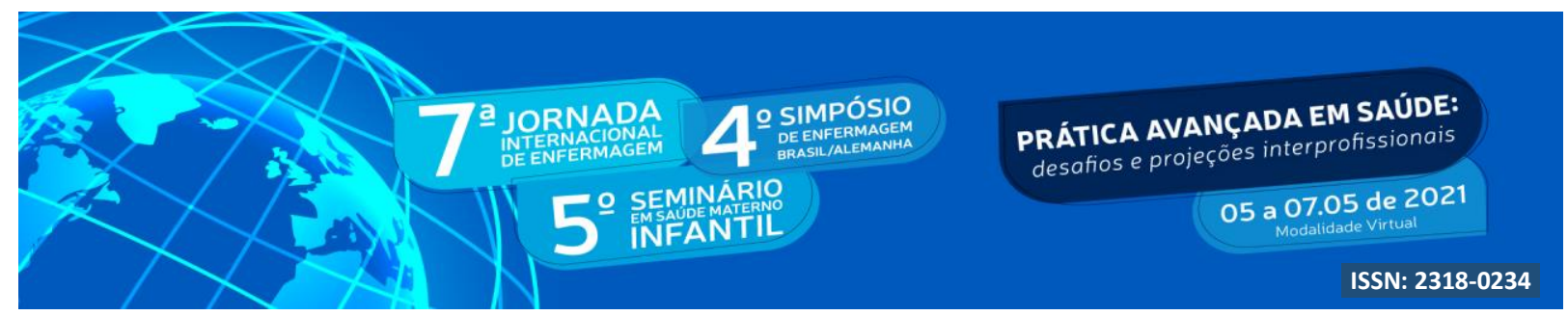

DOI: http://doi.org/10.48195/jie2021-141

\title{
POSSÍVEIS DETERMINANTES NA GRAVIDADE DE PACIENTES HOSPITALIZADOS COM DIFERENTES TIPOS DE ACIDENTE VASCULAR CEREBRAL
}

\section{Francisco Daroda Dutra ${ }^{1}$ Mairin Schott $^{2}{\text { Clandio Timm } \text { Marques }^{3} \text { Elisângela Colpo }}^{4}$}

\begin{abstract}
RESUMO
Objetivou-se comparar características sóciodemográficas, presença de comorbidades e mortalidade em pacientes hospitalizados com Acidente Vascular Cerebral isquêmico (AVCi) ou Acidente Vascular Cerebral hemorrágico (AVCh). Estudo realizado em hospital público da região central do Rio Grande do Sul, composto pacientes hospitalizados com AVC, de ambos os gêneros. Analisou-se o tipo de AVC, doenças associadas, idade, gênero e mortalidade a partir da análise de dados em prontuários. Participaram do estudo 94 pacientes com AVC, predominando AVCi 73,4 \% (n=69), gênero masculino (62\%; $\mathrm{n}=58)$ e idosos $(69 \% ; \mathrm{n}=65)$. Pacientes com AVCi apresentaram maior número de diabéticos quando comparados com AVCh ( $\mathrm{P}=0,029)$. Quase a metade dos pacientes que sofre AVCh $48 \%(\mathrm{n}=12)$ vão a óbito contra apenas 20,6\% $(\mathrm{n}=14)$ dos pacientes com AVCi $(\mathrm{P}=0,009)$. O AVCh tem maior índices de mortalidade quando comparado ao AVCi e ocorre predominantemente em homens, idosos, independentemente do tipo de AVC.
\end{abstract}

Palavras-chave:AVC; Diabetes Mellitus(DM); Hospitalização; Glicemia; Mortalidade.

\begin{abstract}
The objective of this study was to compare sociodemographic characteristics, presence of comorbidities and mortality in patients hospitalized with ischemic stroke (IS) or hemorrhagic stroke (SHA). Study conducted in a public hospital in the central region of Rio Grande do Sul, composed of patients hospitalized with stroke, of both genders. The type of stroke, associated diseases, age, gender and mortality was analyzed from the analysis of data in medical records. The study included 94 patients with stroke, predominantly $73.4 \%(n=69)$, male $(62 \% ; n=58)$ and elderly $(69 \% ; n=65)$. Patients with IS had a higher number of diabetics when compared to SHA $(\mathrm{P}=0.029)$. Almost half of the patients who suffer SHA 48\% ( $n=12)$ die against only $20.6 \%(n=14)$ of patients with IS $(\mathrm{P}=0.009)$. The SHA has higher mortality rates when compared to IS and occurs predominantly in men, elderly, regardless of the type of stroke.
\end{abstract}

Key Words: Stroke; Diabetes Mellitus; Hospitalization; Glycemia; Mortality.

\footnotetext{
${ }^{1}$ Estudante do Curso de Nutrição. Universidade Franciscana -UFN. E-mail: franciscodaroda@ gmail.com

${ }^{2}$ Nutricionista pela Universidade Franciscana. Doutoranda em Ciências Biológicas: Bioquímica Toxicológica pela UFSM. E-mail: mairinschott@ hotmail.com

${ }^{3}$ Professor do curso de Matemática e do Mestrado em Ciências da Saúde e da Vida. Universidade Franciscana -

UFN. E-mail: clandiomarques@gmail.com

${ }^{4}$ Professora dos cursos de Nutrição e do Mestrado em Ciências da Saúde e da Vida. Universidade Franciscana -

UFN. E-mail: elicolpo@ufn.edu.br
} 


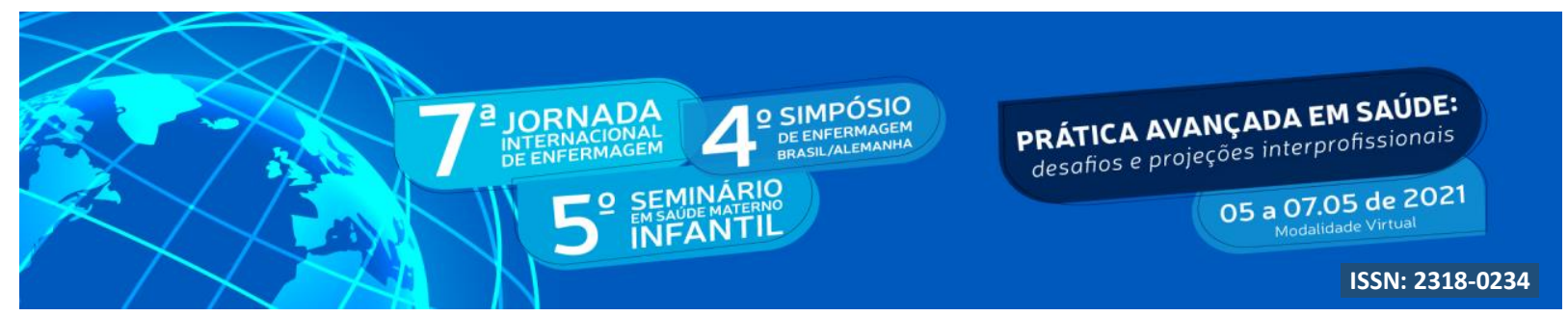

\section{INTRODUÇÃO}

O Acidente Vascular Cerebral (AVC) é a segunda maior causa de morte no mundo e na América do Sul segundo a Organização Mundial da Saúde (OMS, 2016). Diferencia-se em AVC isquêmico e hemorrágico o qual pode subdivir-se em intracerebral e subaracnóide. Estima-se que o AVC atinja 2,26 milhões de pessoas anualmente no Brasil, com essa população sendo majoritariamente constituída por indivíduos idosos de baixa escolaridade, com inadequado estilo de vida e resindindo em sua maioria em centros urbanos (BENSENOR et al., 2016).

Múltiplas comorbidades como: Diabetes Mellitus (DM), Hipertensão Arterial Sistêmica (HAS), tabagismo, Fibrilação Atrial (FA), Dislipidemia, Obesidade e uso de álcool associamse a menor sobrevivência, acesso a reabilitação e medicação influenciando a mortalidade após AVC (CASTRO et al., 2017). Além de serem relacionadas a diferentes tipos de AVC, hábitos nocivos como o tabagisto associa-se a maior incidência de AVC subaracnóide em mulheres, enquanto mulheres diabéticas possuem maior risco para AVCi e AVCh Intracerebral (PRICE et al., 2018).

Multimorbidades são características da transição demográfica, em constante crescimento o DM aumentou a prevalência em 30,1\% entre 2007 e 2017 para o DM, tornando a terceira maior causa de desabilidade para homens no mundo de acordo com Kyu et al. (2018). O que corrobora para a maior incidência de AVC e maiores taxas de mortalidade em indivíduos diagnosticados com DM e socioeconomicamente desfavorecidos (CASTRO et al., 2017; MARSHALL et al., 2015).

No Brasil, HAS e dislipidemia seguem-se como principais fatores de risco para o AVC (MARRONE et al., 2013). Entretanto, o DM além de aumentar as chances de risco para o AVC, corrobora para a hiperglicemia durante o evento, o que relaciona-se a piores prognósticos (LUITSE et al., 2012). A presença de Diabetes Mellitus tipo 2 (DM2) pode ser um preditor para AVC Intracerebral ou Isquêmico (LARSSON et al., 2018).

A gravidade está diretamente relacionada ao gênero e faixa etária, com homens de média idade presentes em maior número nas ocorrências, as quais seguem aumentando para essa população (CABRAL et al., 2017). Além disso, a progressão da idade reforça a incidência e a 


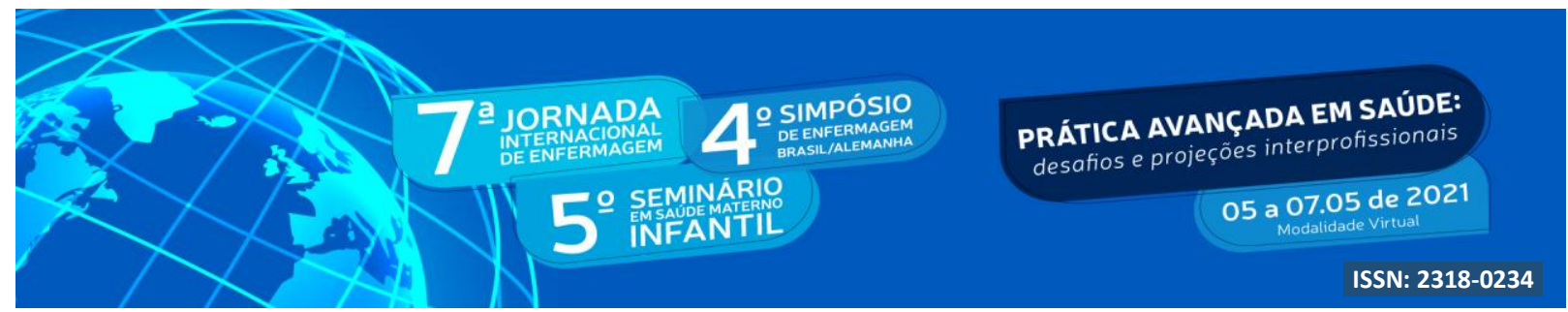

letalidade do AVC em hospitais públicos do Brasil no período de 2009 a 2016 (DANTAS et al., 2016).

Não obstante, a incidência do AVC segue em crescimento no país, conjuntamente à expectativa de vida (JAMES et al., 2018), o que acentua a necessidade de reduzir a chance para tais comorbidades e a possibilidade de um AVC ocorrer, subretudo reduzir também a chance de viver-se mais anos com desabilidade e atenuar a gravidade em decorrência de ambos os tipos de AVC. Com isso, o objetivo deste estudo foi comparar características sóciodemográficas, presença de comorbidades e mortalidade em pacientes hospitalizados com AVC isquêmico (AVCi) ou hemorrágico (AVCh).

\section{METODOLOGIA}

O trabalho possui delineamento transversal, quantitativo. A pesquisa foi realizada em um hospital público da região central do Rio Grande do Sul, Brasil, durante os meses de agosto 2018 a agosto de 2019. A amostra foi por conveniência, composta por adultos e idosos hospitalizados com diagnóstico de AVC, de ambos os gêneros. O estudo foi aprovado pelo Comitê de Ética e Pesquisa e está registrado sob o número 1.579.090, conforme a Resolução n. 466/12 do Conselho Nacional de Saúde, referente a pesquisas com seres humanos. O termo de consentimento livre e esclarecido (TCLE) foi obtido por escrito de todos os participantes.

Foram incluídos pacientes que tiveram o diagnóstico de AVC confirmado por tomografia ou ressonância magnética, realizado pelo médico e coletado dos prontuários, além disso, foram subdivididos em AVCi e AVCh.

Os seguintes dados de identificação pessoal dos pacientes foram coletados do prontuário: gênero, idade, doenças associadas, medição de glicemia capilar (HGTs), realizado conforme prescrição médica e dados relacionados a mortalidade dos pacientes como alta hospitalar ou óbito.

Inicialmente foi aplicado o teste de normalidade de Kolmogov-Smirnov para as variáveis quantitativas. Estas foram apresentadas como média e desvio padrão, e frequência relativa conforme a distribuição dos dados. $\mathrm{Na}$ análise em relação as variáveis qualitativas foram aplicadas o teste do qui-quadrado. Em relação aos valores da glicemia comparando os grupos dos pacientes com AVCi e AVCh foi utilizado o teste de Mann-Whitney. O nível de 


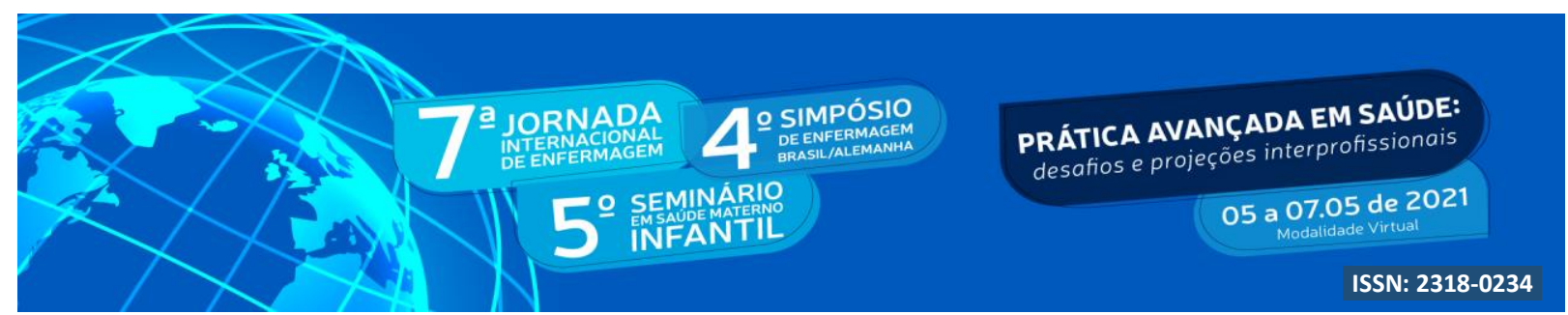

significância foi estabelecido para um valor de $\mathrm{p}<0,05$. O software IBM SPSS Versão 25 foi utilizado como ferramenta computacional para a análise estatística dos dados.

\section{RESULTADOS E DISCUSSÃO}

A amostra total do estudo foi composta por noventa e quatro participantes hospitalizados pós Acidente Vascular Cerebral (AVC), sendo 73,4 \% (n=69) com AVCi e 26,6 \% (n=25) com AVCh. Dos participantes, a maioria era do gênero masculino e idosos, sendo a média de idade dos pacientes com AVC isquêmico de 65,3 $\pm 11,5$ anos;

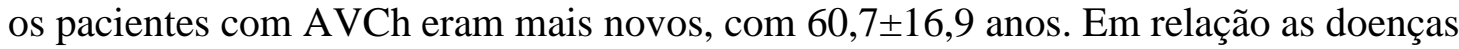
associadas, a maioria dos pacientes com AVC apresentaram Hipertensão Arterial Sistêmica (HAS). A presença de Diabetes Mellitus foi maior estatisticamente nos pacientes com AVCi em relação ao AVCh, conforme Tabela 1.

Tabela 1: Caracteríticas dos pacientes hospitalizados com diferentes tipos de AVC.

\begin{tabular}{lccc}
\hline \multicolumn{5}{c}{ Tipo de AVC } \\
\hline \multicolumn{5}{c}{ Variáveis } & Isquêmico & Hemorrágico & $\mathrm{p}$ \\
\hline Gênero & & \\
\hline Masculino & $40(58)$ & $18(72)$ & 0,216 \\
\hline Feminino & $29(42)$ & $7(28)$ & \\
\hline Idade & & \\
\hline Adulto & $20(29)$ & $9(36)$ & 0,515 \\
\hline Idoso & $49(71)$ & $16(64)$ & \\
\hline Diabetes Mellitus & & \\
\hline Sim & $34(49,3)$ & $6(24)$ & \\
\hline Não & $35(50,7)$ & $19(76)$ & \\
\hline HAS & & & \\
\hline Sim & $40(58)$ & $17(68)$ & 0,379 \\
\hline Não & $29(42)$ & $8(32)$ & \\
\hline
\end{tabular}

HAS: Hipertensão Arterial Sistêmica. Valores expressos em n (\%). Teste qui-quadrado. $* \mathrm{P}<$ 0,05 .

Em nossa amostra, prevaleceu-se o público masculino como maior acometido pelo evento cerebrovascular, sendo 58 homens e 36 mulheres. Em acordo a análise de Barker-collo et al. (2015) onde há predomínio do gênero masculino para incidência de 


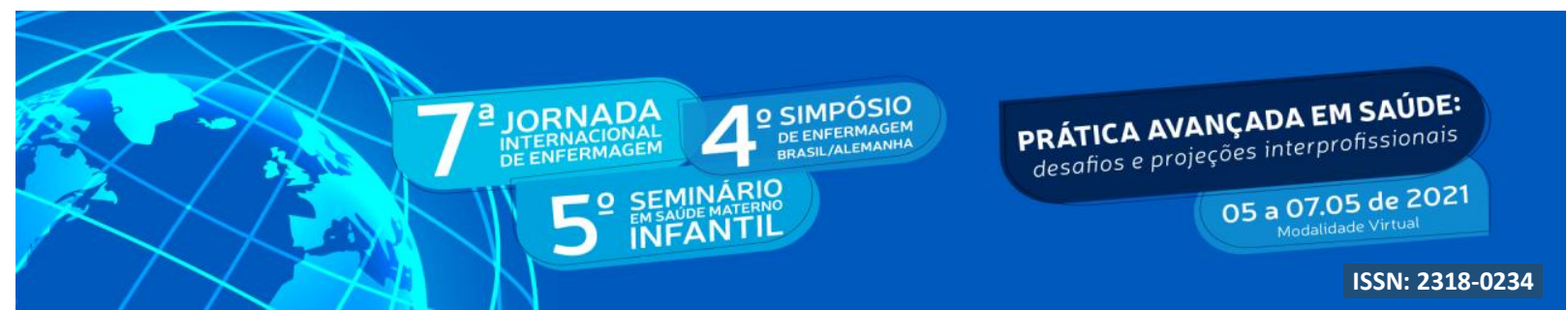

AVC em diferentes locais do mundo. Não obstante, embora homens sofram mais do evento cerebrovascular hemorrágico, que mulheres, não verificou-se diferença significativa entre o gênero e o tipo de $\operatorname{AVC}\left(\chi^{2}=1,528 ; p=0,216\right)$.

Logo, verificou-se existir uma associação estatisticamente significativa entre o paciente ter ou não Diabetes Mellitus e o tipo de $\operatorname{AVC}\left(\chi^{2}=4,796 ; p=0,029\right)$. Corroborando aos achados de Larsson et al. (2018), que aponta para a presença de DM2 como um fator de risco para AVC por artérias maiores, que relaciona-se ao AVC isquêmico e intracerebral. Em conjunto com os achados de Price et al. (2018), tal qual há menor risco para um evento hemorrágico subaracnóide em pacientes DM.

Quando analisado as glicemias dos pacientes, observou-se que indivíduos com AVCi apresentaram hiperglicemias (162,9 $\pm 58,1 \mathrm{mg} / \mathrm{dL})$. Conforme Bruno et al. (2004) as hiperglicemias são mais comumente encontradas em pacientes DM. Os pacientes com AVCh apresentaram menores médias glicêmicas (138,8 $\pm 40,7 \mathrm{mg} / \mathrm{dL})$, sendo caracterizadas em normoglicemia de acordo com a SBD, 2014 conforme Figura 1. No entanto, não foram observadas diferenças estatísticas entre as médias glicêmicas $(\mathrm{P}=$ $0,23)$.

Não obstante, a gravidade do AVC está relacionada a maiores glicemias (TZIOMALOS et al., 2017). Que podem ser derivadas do estresse ou da presença de DM prévio (LUITSE et al., 2012). Sendo o DM prévio e a hiperglicemia preditores para eventos isquêmicos ou hemorrágicos intracerebrais (VAN SLOTEN et al., 2020). 

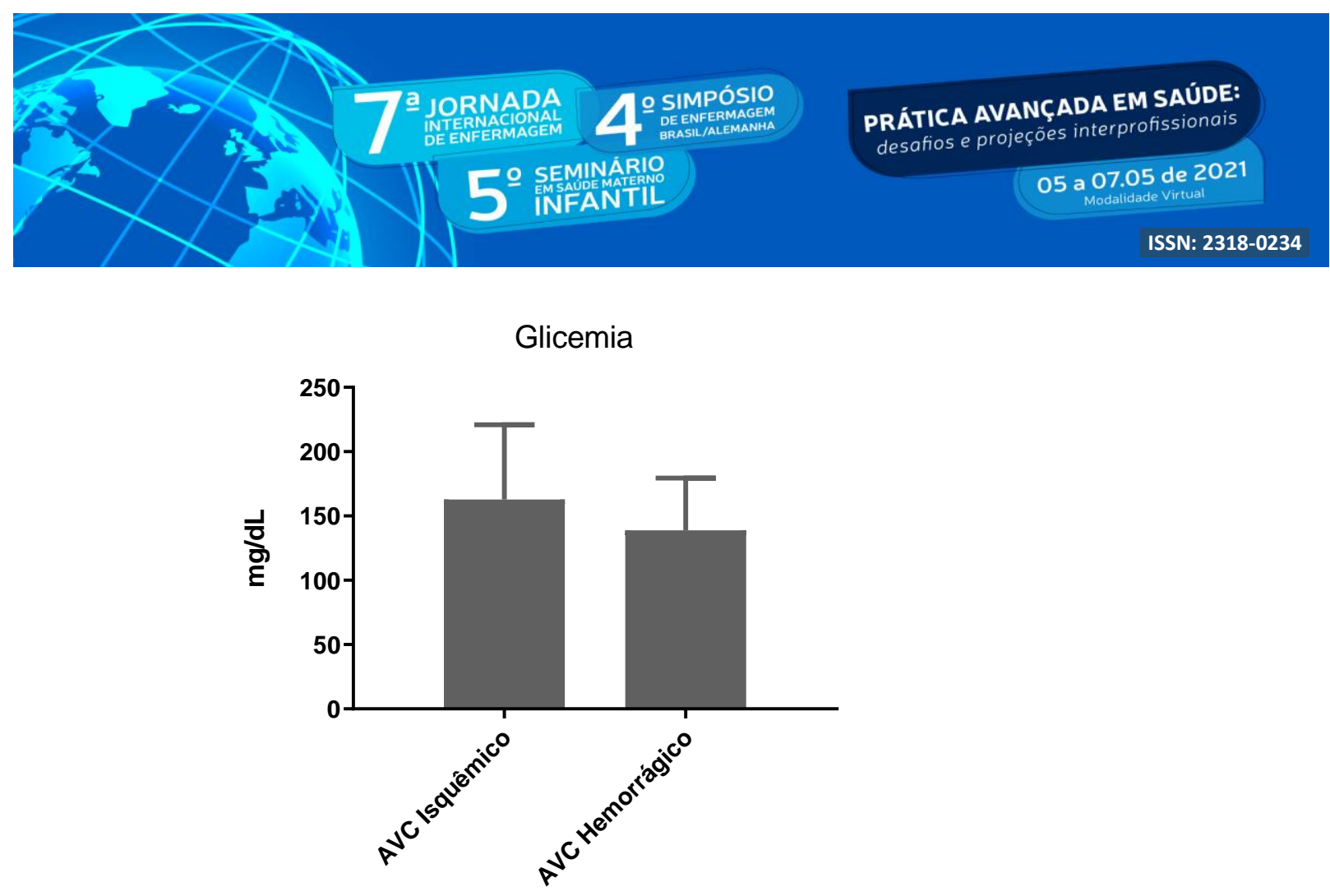

Figura 1: Médias das glicemias conforme diferentes tipos de AVC. Valores expressos em média \pm desvio-padrão.

Além disso, foi avaliado a gravidade dos pacientes a partir de dados sobre a mortalidade dos pacientes. Foi observado que $48 \%(n=12)$ dos pacientes com AVCh foram a óbito enquanto esse percentual foi de 20,6\% $(n=14)$ nos pacientes com AVC isquêmico, dados estatisticamente significativos $\left(\chi^{2}=6,820 ; p=0,009\right)$, conforme Figura 2. Achado de acordo com o estudo de Gonzalez-Perez et al. (2013), que demonstra chance de mortalidade para AVCh maior que um terço, independente do subtipo, se subarácnóide ou intracerebral. 

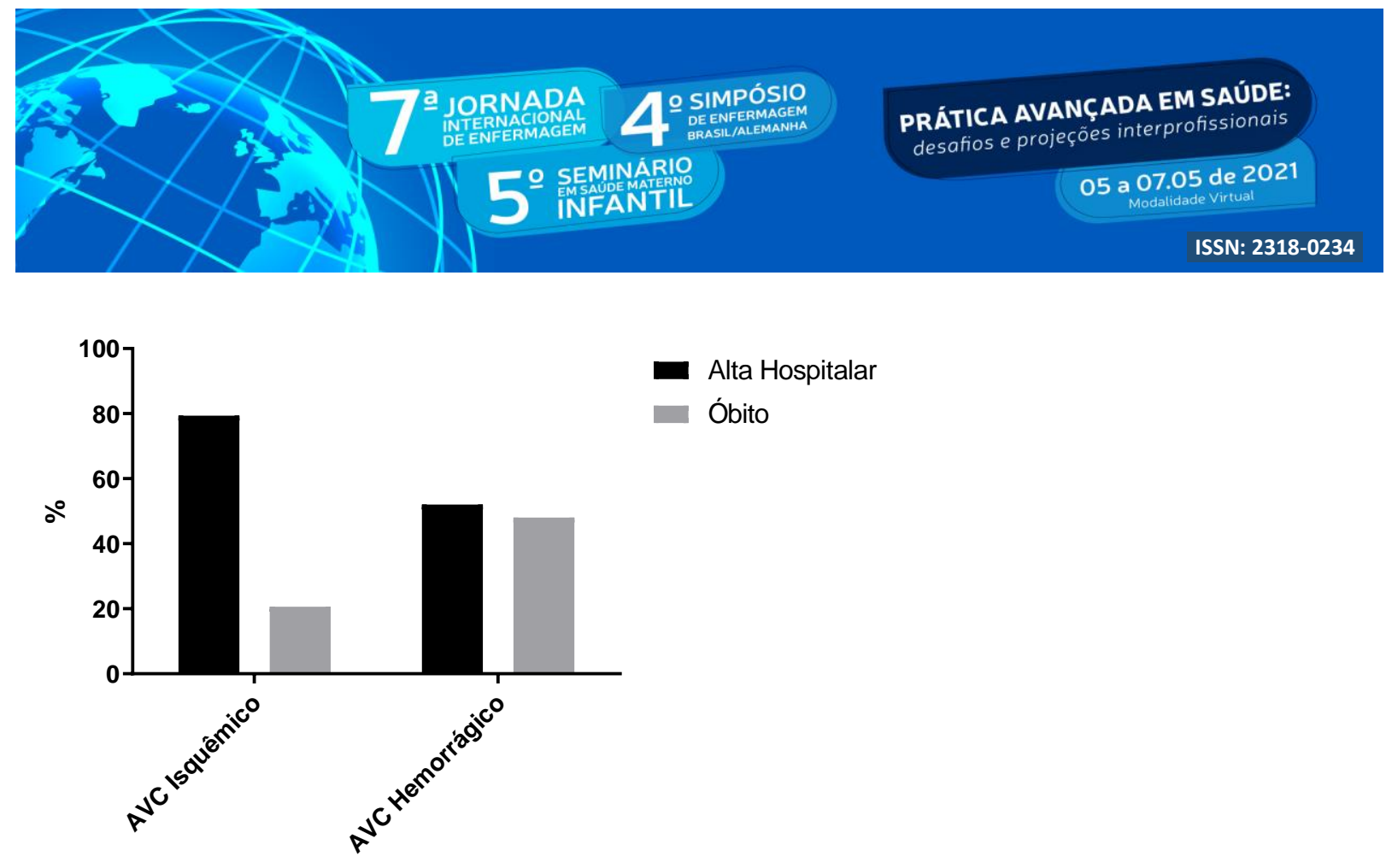

Figura 02: Mortalidade dos pacientes conforme diferentes tipos de AVC. Valores expressos em percentual.

\section{CONCLUSÃO}

Em nossa amostra podemos concluir que existe um maior predomínio de pacientes do gênero masculino e idosos, tanto no AVCi como no AVCh. A presença de DM prévio relacionou-se mais com o AVC isquêmico, bem como os eventos hiperglicêmicos. No entanto, houve um maior predomínio de óbitos em pacientes com AVCh. Mais estudos são necessários a fim de determinar outras medidas que possam identificar diferentes características entre eventos isquêmicos ou hemorrágicos nesses pacientes.

\section{REFERÊNCIAS}

BARKER-COLLO $S$. et al. Diferenças entre os sexos na incidência, prevalência, mortalidade e anos de vida ajustados por incapacidade: resultados do Global Burden of Disease Study 2013. Neuroepidemiology, 2015.

BENSENOR, I. M. et al. Prevalência de AVC e deficiência associada no Brasil: Pesquisa Nacional de Saúde - 2013. Arquivos de Neuro-Psiquiatria, 2015.

BRUNO. A. et al. Qual a importância da hiperglicemia durante o infarto cerebral agudo? The Neurologist, 2004. 


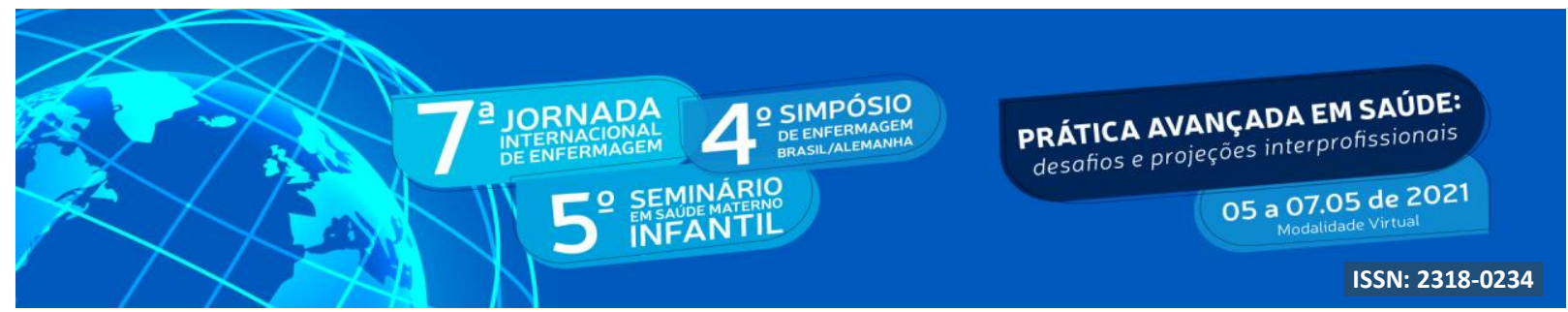

CABRAL, N. L. et al. Aumento da incidência de AVC em jovens adultos em um país de renda média. Stroke, 2017.

CASTRO H, H, G. et al. Multimorbidades estão associadas à menor sobrevida no AVC isquêmico: resultados de uma coorte brasileira de AVC (Estudo EMMA). Cerebrovasc Dis, 2017.

DANTAS, L. F. et al. Internações públicas por acidente vascular cerebral no Brasil de 2009 a 2016. PLoS ONE, 2019.

Diretrizes Sociedade Brasileira do Diabetes. Métodos e critérios para diagnóstico do diabetes mellitus, 2014.

GONZALEZ-PEREZ, A. et al. Mortalidade após AVC hemorrágico: Dados da prática geral (Rede de Melhoria da Saúde). Neurology, 2013.

JAMES S. L., et al. Incidência global, regional e nacional, prevalência e anos vividos com deficiência por 354 doenças e lesões em 195 países e territórios, 1990-2017: uma análise sistemática para o Global Burden of Disease Study 2017. Lancet, 2018.

KYU, H. H. et al. Anos de vida ajustados por deficiência globais, regionais e nacionais (DALYs) para 359 doenças e lesões e expectativa de vida saudável (HALE) para 195 países e territórios, 1990-2017: uma análise sistemática para o Global Burden of Disease Study 2017. Lancet, 2018.

LARSSON, S. C. et al. Diabetes tipo 2, glicose, insulina, IMC e subtipos de AVC isquêmico. Neurology, 2018.

LUITSE, M. J. A. et al. Diabetes, hiperglicemia e acidente vascular cerebral isquêmico agudo. The Lancet Neurology, 2012.

MARRONE LC. et al. Fatores de risco entre subtipos de AVC no Brasil. Journal of Stroke and Cerebrovascular Diseases, 2013.

MARSHALL, L. J. al. Os efeitos do status socioeconômico no risco e nos resultados de AVC. The Lancet Neurology, 2015.

PRICE, A. J. Et al. Diferenças nos fatores de risco para 3 tipos de AVC. Neurology, 2018. TZIOMALOS, K. et al. Hiperglicemia de estresse e derrame isquêmico agudo no hospital, Metabolism, 2017.

VAN SLOTEN. T. T. et al. Complicações microvasculares cerebrais do diabetes tipo 2: acidente vascular cerebral, disfunção cognitiva e depressão. The Lancet Diabetes \& Endocrinology, 2020. 